\title{
Effect of artemether-lumefantrine treatment of falciparum malaria on urogenital schistosomiasis in co-infected School Aged Children in North Central of Nigeria
}

\author{
Ayodele Adeyemi ADEDOJA*, Ajibola Aliu AKANBI and Adebola John OSHODI \\ Department of Medical Microbiology \& Parasitology, University of Ilorin Teaching Hospital, \\ Ilorin. Nigeria. PMB 1459, Ilorin, Nigeria. \\ *Corresponding author, E-mail: ayodeleadedoja@gmail.com ; Tel: +2347037986672
}

\begin{abstract}
The focus of the present study is to assess the effect of artemether-lumefantrine treatment of falciparum malaria on urogenital schistosomiasis in co-infected individuals. Urine samples were collected from 159 microscopically confirmed malaria patients and diagnosed for urogenital schistosomiasis before treatment. The schistosomiasis cure rate and egg reduction were determined in co-infected patients, who were treated with artemether-lumefantrine. Out of 103 malaria infected children, 56 were co-infected $54.4 \%$ (56/103) with schistosomiasis. All 56 co-infected patients were found urine-negative for Schistosoma haematobium eggs four weeks after treatment. The extent of co-infection was associated with age and sex level. Cure rate and egg reduction rate following the treatment of artemether-lumefantrine were $100 \%(\mathrm{p}=0.0000)$. Artemetherlumefantrine was effective against $S$. haematobium in co-infected children. Further studies however, are needed for a better understanding of the efficacy of artemether lumefantrine against schistosome infection with ranges of intensity.

() 2014 International Formulae Group. All rights reserved.
\end{abstract}

Keywords: Urogenital haematobium, malaria, Cure rate, Egg reduction rate.

\section{INTRODUCTION}

Malaria is seen in all countries extending from $40^{\circ} \mathrm{S}$ and $60^{\circ} \mathrm{N}$ of equator covering a large portion of tropical and subtropical region (Suthar et al., 2013). It is a public health problem in many countries. According to the estimates of WHO, there were about 219 million cases of malaria in 2010 (with an uncertainty range of 154 million to 289 million) and an estimated

660000 deaths (with an uncertainty range of 490000 to 836000$)$ (WHO, 2013). Plasmodium falciparum is responsible for a high burden of disease and a loss of growth in endemic countries estimated to be as high as $1.3 \%$ of gross domestic product per year (Ojurongbe et al., 2011). The attack of malaria continues through at least the first 5 years of life before most children living in endemic regions develop immunity sufficient to suppress severe pathogenesis (Baird, 1998).

In malaria endemic areas, co-infection with multiple parasites, including Schistosoma species, is common (Ojurongbe et al., 2011). Malaria and urogenital schistosomiasis are parasitic diseases causing high morbidity and 
mortality in most tropical parts of the world, where climatic conditions and sanitation practices favours their prevalence. It is estimated that over a third of the world's population, mainly those individuals living in the tropics and subtropics, are infected by parasitic helminthes or one or more of the species of Plasmodium (Snow et al., 2005). Praziquantel is the drug of choice for the treatment of schistosomiasis. Although no resistance to praziquantel has been described, low cure rates of schistosomiasis have been reported (Doenhoff et al., 2008). There have been report of resistant to praziquantel from Egypt suggesting that the worms are in some way less responsive to the drug hence alternative is desired (Cioli and PicaMattoccia, 200; William, 2001).

Artemisinin based combination therapy was introduced in Nigeria in 2005 as the first-line anti-malarial drug $(\mathrm{FMOH}$, 2005). Studies have reported the beneficial effects of treating malaria on schistosomiasis in coinfected individuals (Abay et al., 2013). Outside Africa studies have been done to study the beneficial effect of malaria treatment with artemether-lumefantrine in individuals infected with the both infections. Song et al. (1998), reported the promising effect of artemether- lumefantrine on controlling acute schistosomiasis and reducing the infection rate in a study in schistosomiasis endemic area of Poyang Lake, Jiangxi Province of China (Song et al., 1998). Xiao and Catto (1989), in their study reported activities of artemether against S. mansoni. Though different studies have documented the prevalence of falciparum malaria and urogenital schistosomiasis co-infections in different settings in Africa; in Nigeria (Ojurongbe et al., 2011), Zambia (Rutagwera and Tylleskär, 2012), and Ethiopia (Abay et al., 2013). But there are fewer reports on the effectiveness of falciparum malaria treatment with artemether- lumefantrine in subjects coinfected with $S$. haematobium. The aim of this study is to determine the beneficial effect of artemether-lumefantrine treatment on urogenital schistosomiasis in children coinfected with $P$. falciparum malaria at a dosage regimen used for the treatment of $P$. falciparum malaria in school aged children in
Pategi local Government Area of Kwara State, in the North Central of Nigeria.

\section{MATERIALS AND METHODS}

The study area and population

Pategi is the capital of Pategi Local Government located in Pategi Local Government Area of Kwara State, Nigeria. Population of Pategi is 110,852 people (National Population Commission of Nigeria). The town falls into stable malaria transmission zone where malaria is present throughout the year with a marked increase during the raining season which normally runs from April to September. The town stands on higher level and the soil can be described as well drained, moderately leached and with moderate humus content. Major occupations include farming (rice farming), fishing and petty trading. The town has a general hospital owned by state government and many private clinics. The study was carried out from March to May 2013, which spanned through wet season. Children were recruited from three schools consisting of a nursery and two primary schools with age range of 1 to 15 years. Random sampling method was used to collect specimens from the children. The inclusion criteria for the study included: (1) children aged 1-15 years; (2) parents or guardians gave consent; and (3) children lived in the study area. Before samples were collected, demographic data such as sex, age, and name of subject were recorded.

\section{Ethical Issues}

Ethical clearance for the study was obtained from the Kwara State Ministry of Health Ethical Committee, Nigeria (MOH/KS/777/41) and from the Local Government Primary School Board, and the parents. The study was explained to the school heads, parents, wards before specimen collection.

\section{Detection and quantification of malaria parasites}

Finger-prick blood samples were obtained for slide microscopy. Blood smears were air-dried, stained with $4 \%$ Giemsa and analysed under a light microscope $(\times 100$ oil immersion) to detect asexual forms of $P$. 
falciparum. A result was considered negative if no parasites were detected. Each slide was read independently by two study technicians. In the case of a discrepant qualitative result (negative or positive), a third reading was done by the designated technician. The infected children were treated accordingly with the help of medical staff in the general hospital.

Urine samples were analysed using sedimentation method as described by (Okoli et al., 2006). Briefly samples were left to stand on the bench for about $30 \mathrm{~min}$. Afterwards the topmost part of the urine was discarded leaving about $10 \mathrm{~mL}$ in the bottle. The content of each bottle was mixed thoroughly with the sediment and was transferred into a $20 \mathrm{ml}$ centrifuge tube. The tubes were then centrifuged at 1000 revolution per minute for $2 \mathrm{~min}$. The supernatant was discarded and the residue was put on a clean glass slide and examined under $10 \mathrm{X}$ objective lens of the microscope. Intensity of infection was estimated as number eggs per $10 \mathrm{ml}$ of urine.

\section{Statistical analysis}

Statistical analysis was done using SPSS version 10 for windows. For analysis, prevalence of malaria, and urinary schistosomiasis were compared using $\chi^{2}$ tests. Two sided $p$ values $<0.001$ indicated statistical significance.

\section{RESULTS}

A total of 159 pupils were recruited into the study. The mean age was 8.35 years
( $\mathrm{SD} \pm$ 2.747), male: female is $81 / 78$. The prevalence of $P$. falciparum was $(64.8 \%)$ while the prevalence of urogenital schistosomiasis was $(35.2 \%)$ and co-infection was $54.4 \%$.

Urogenital haematobium egg load and $P$. falciparum co-infection stratified by sex and age of the children is shown in Table 2. Female children were more infected with $P$. falciparum $(69.2 \%)$ than male children $(60.5 \%)$. ( $\mathrm{P}=0.249)$. The percentage coinfection is $54.4 \%$ out of which female children were more co-infected with $P$. falciparum and S. haematobium (53.6\%) than male children $(46.4 \%)$. Moderate and light coinfection was observed in females $(66.7 \%)$ and $(33.3 \%)$ respectfully. Children of age group 7-9 years were more infected with $P$. falciparum $39(37.9 \%)$ followed by age group 10-12 years $32(31.1 \%)$. In S. haematobium category, children of age group 7-9 years were more co-infected with $S$. haematobium $28(50.0 \%)$ followed by age group 4-6 years $14(25.0 \%)$. Highest moderate infection $19(51.4 \%)$ was observed in age group 7-9 years and lowest light $S$. haematobium infection $1(5.3 \%)$ was seen in age group 1315 years. $(\mathrm{P}=0.000)$

Effect of artemether-lumefantrine on $S$. haematobium in falciparum malaria coinfected subjects shown in Table 3. All the pupils positive for falciparum malaria and $S$. haematobium were treated with artemetherlumefamentrine. None of the pupils passed egg of S. haematobium after four weeks post treatment. The curative rate was $(100.0 \%)$.

Table 1: Enrollment data of the subjects recruited into the study.

\begin{tabular}{lc}
\hline Number of subjects examined & $\mathbf{1 5 9}$ \\
\hline Mean age (yrs) \pm SD & $8.35 \pm 2.747$ \\
Sex (male/female) & $81 / 78$ \\
P. falciparum (Number positive) & $103(64.8 \%)$ \\
Schistosoma haematobium & $56(35.2 \%)$ \\
P. falciparum, and Schistosoma haematobium coinfection & $54.4 \%$ \\
\hline
\end{tabular}


Table 2: Urogenital haematobium egg load and P.falciparum co-infection sratified by sex and age of the children.

\begin{tabular}{|c|c|c|c|c|c|c|c|}
\hline Variables & Sub category & $\begin{array}{c}\text { FreqN=159 } \\
(\%)\end{array}$ & $\begin{array}{c}\text { No Positive } \\
\text { P.falciparum }(\%)\end{array}$ & $\begin{array}{c}\text { Number Positive } \\
\text { co-infection } \\
\mathrm{N}=56(\%)\end{array}$ & $\begin{array}{c}\text { Light infection <50 } \\
(\text { eg/10mL) }(\%)\end{array}$ & $\begin{array}{c}\text { Moderate } \\
\text { infection }>50 \\
(\text { eg/10mL }) \\
(\%)\end{array}$ & $\begin{array}{c}\text { Over all } \\
\% \text { Co-infection }\end{array}$ \\
\hline \multirow[t]{3}{*}{ Sex } & Male & $81(50.9)$ & $23(14.5)$ & $26(46.4)$ & $9(34.6)$ & $17(65.4)$ & $(54.4)$ \\
\hline & Female & $78(49.1)$ & $33(20.8)$ & $30(53.6)$ & $10(33.3)$ & $20(66.7)$ & \\
\hline & & & $\mathrm{N}=103$ & & $\mathrm{~N}=19$ & $\mathrm{~N}=37$ & \\
\hline \multirow[t]{5}{*}{ Age } & $<4$ & 1 & $1(0.9)$ & $0(0.0)$ & $0(0.0)$ & $0(0.0)$ & \\
\hline & $4-6$ & 31 & $26(25.2)$ & $14(25.0)$ & $6(31.6)$ & $8(21.6)$ & Chi Sqr=2.189 \\
\hline & $7-9$ & 31 & $39(37.9)$ & $28(50.0)$ & $9(47.4)$ & $19(51.4)$ & Pvalue $=0.000$ \\
\hline & $10-12$ & 37 & $32(31.1)$ & 11(19.6) & $3(15.8)$ & $8(21.6)$ & \\
\hline & $13-15$ & 3 & $5(4.9)$ & $3(5.4)$ & $1(5.3)$ & $2(5.4)$ & \\
\hline
\end{tabular}

Table 3: Effect of artemether-lumefantrine on urogenital haematobium in falciparum malaria co-infected subjects after four weeks post treatment.

\begin{tabular}{lccc}
\hline Variables & Pretreatment & Post treatment & P value* \\
\hline Schistosomiasis cases & 56 & 0 & 0.000 \\
Cure rate $(\%)$ & 100 & 0.000 \\
Overall $(\mathrm{eg} / 10 \mathrm{~mL})$ & & & \\
Egg load reduction $(\%)$ & & 100 & \\
\hline
\end{tabular}




\section{DISCUSSION}

The results of the present study showed that falciparum malaria and urogenital schistosomiasis are of public health importance among school children in schools aged children in Pategi, Kwara State, Nigeria. The prevalence of $P$. falciparum malaria observed in this study was higher more than the prevalence observed elsewhere both locally and internationally; Nwaorgu and Orajaka (2011), recorded 58.2\% in South East Nigeria, Kimbi et al. (2013), recorded $33.8 \%$ in Cameroun. This prevalence supported is in agreement with the view that majority of malaria infections in individuals living in endemic regions are non clinical with the young children bearing the highest burden of disease and carrying non clinical infections for most of the time (Ojurongbe et al., 2011).

However, the prevalence of $35.2 \%$ for Schistosoma haematobium observed in this study was not consistence with prevalences reported elsewhere. Ologunde et al. (2012), reported a higher prevalence of $75.6 \%$ in Ogbese Ekiti in Nigeria while Rutagwera and Tylleskär (2012), reported a lower prevalence of $29.5 \%$ Zambezi District of Zambia.

The current study has established that Pategi town in Pategi Local Government is endemic for both $P$. falciparum and $S$. haematobium infections. The co-infection rate of $54.4 \%$ observed in the present study was similar to the prevalent rate reported by Amuta and Houmsou (2014), who reported a prevalent rate of $(55.0 \%)$ in an investigation from Guma Local Government Area of Benue State Nigeria.

Females have been shown to have higher co-infection rates compared with males. The observed differences among females and males might probably be exposure related. Females help in daily chores, fetching water from the polluted stream, helping on the farm in addition to swimming and adventures during the holidays. The town is situated along the river Niger bank. Hence fishing and local rice production are the occupations of the parents.
Swampy rice is produced both as food and cash crop. Swampy rice is planted in the water logged soil and this provides contact with infected water.

The current findings also revealed that the 7-9 year old group had the highest co-infection rate $(37.9 \%)$. In the current study, the co-infection rate increased as age increased, peaked at age group 7-9 years and then decreased. The difference in coinfection positivity in the age group was significant $(\mathrm{P}=0.0000)$. The decrease in coinfection rate from age 7-9 may have resulted from repeated exposure to infection which might have increased the immunity response against the infections as it has been reported elsewhere by Mazigo et al. (2010).

Artemether-lumefantrine was introduced in Nigeria for the treatment of Plasmodium falciparum infection in 2005 Federal Ministry of Health (FMOH, 2005). From experimental studies, artemisinin derivatives act against other parasites, as well as against tumor cells (Jacob et al., 2006). Hence, it is cogent to investigate the clinical side benefits of artemisinins when used in the treatment of malaria especially in environment endemic for both parasites. The focus of this present study is to find out the beneficial effect of artemether-lumefantrine on urogenital schistosomiasis when used to treat falciparum malaria in children infected with both parasitic infections. The results after a month check up revealed that all coinfected children did not have eggs of $S$. haematobium in their urine following artemether lumefantrine therapy at doses used for the treatment of malaria. This is comparable to the results of a study conducted in Sudan and Ethiopia, in which malaria patients treated with artemetherlumefantrine did not excrete $S$. mansoni eggs after a month check-up (Adam et al., 2008; Abay et al., 2013) and also in the Côte d'Ivoire (Utzinger et al., 2000). The cure rate of schistosomiasis in the present study was $100 \%(p=0.000)$. The result of this study is also in support of the outcome of the study of Xiao and Catto (1989) who studied the in 
vitro and in vivo effect of artemether on Schistosoma mansoni.

\section{Conclusion}

Since studies have proven the emergence of Schistosoma species resistance to praziquantel, the desired alternative might be artemisinins derivatives. The findings point to the need for constant and continuous epidemiological studies in areas where malaria and schistosomiasis co-exist and where artemisinin-based combination therapies have been introduced. Since artemisinin-based combination therapy is part of malaria control package in Nigeria, it has benefits against schistosomiasis.

\section{ACKNOWLEDGEMENTS}

The authors are grateful to all parents and guardian who volunteered to participate in the study.

\section{REFERENCES}

Abay SM, Mulugeta T, Nigus F, Abiy H. 2013. Plasmodium falciparum and Schistosoma mansoni coinfection and the side benefit of artemether-lumefantrine in malaria patients. $J$ Infect Dev Ctries, 7(6): 468-474.

Adam I, Elhardello A, Elhadi O, Abdalla E, Elmardi KA, Jansen FH. 2008. The antischistosomal efficacies of artesunatesulfamethoxypyrazine-pyrimethaminc and artemether-lumefantrine administered as treatment for uncomplicated, Plasmodium falciparum malaria. Ann. Trop Med. Parasitol., 102: 39-44.

Amuta EU, Houmsou RS. 2014. Prevalence, intensity of infection and risk factors of urinary schistosomiasis in pre-school and school aged children in Guma Local Government Area, Nigeria. Asian Pacific Journal of Tropical Medicine, 7(1): 3439.

Baird JK. 1998. Age-dependent characteristics of protection v. susceptibility to Plasmodium falciparum. Ann Trop Med Parasitol., 92(4): 367-90.
Boulanger D, Dieng Y, Cisse B, Remoue F, Capuano F, Dieme JL, Ndiaye T, Sokhna C, Trape JF, Greenwood B, Simondon F. 2007. Antischistosomal efficacy of artesunate combination therapies administered as curative treatments for malaria attacks. Trans. $R$. Soc. Trop. Med. Hyg., 101: 113-116.

Cioli D, Pica-Mattoccia L. 2003. Praziqantel. Parasitol Res., 1: 3-9.

Doenhoff M, Cioli D, Utzinger J. 2008. Praziquantel:mechanism of actions resistance and new derivative for schistosomiasis. Curr. Opin. Infec. Dis., 21: 659-667.

Federal Ministry of Health 2005. National Antimalarial Treatment Guidelines. National Malaria and Vector Control Division: Abuja, Nigeria.

Jacob G, Wakninea J, Nicholas M. 2006. Current perspectives on the mechanism of action of artemisinins. Internl. J. Parasitol., 36: 1427-1441.

Keiser J, N'Guessan NA, Adoubryn KD, Silué KD, Vounatsou P, Hatz C, Utzinger J, N'Goran EK. 2010. Efficacy and safety of mefloquine, artesunate, mefloquine-artesunate, and praziquantel against Schistosoma haematobium: randomized, exploratory open-label trial. Clin Infect Dis., 50: 1205-1213.

Kimbi HK, Sumbele IUN, Nweboh M, Anchang-Kimbi JK, Lum E, Nana Y, Ndip LM, Njom H, Lehman LG. 2013. Malaria and haematologic parameters of pupils at different altitudes along the slope of Mount Cameroon: a crosssectional study. Malaria Journal, 12: 193

Mazigo H, Waihenya R, Lwambo N. 2010. Co-infection with Plasmodium falciparum, Schistosoma mansoni and intestinal helminthes among schoolchildren in endemic areas of northwestern Tanzania. Parasit Vectors, 3: 44 .

Nacher M, Singhasivanon P, Treeprasertsuk S, Vannaphan S, Traore B, Looareesuwan S. 2002. Intestinal 
helminths and malnutrition are independently associated with protection from cerebral malaria in Thailand. Ann Trop Med Parasitol., 96(1): 5-13.

Nwaorgu OC. Orajaka, BN. 2011. Prevalence of Malaria among Children 1-10 Years Old in Communities in Awka North Local Government Area, Anambra State South East Nigeria. International Multidisciplinary Journal, Ethiopia, 5(5): 264-281.

Obonyo CO, Muok EMO, Mwinzi PNM. 2010. Efficacy of artesunate with sulfalene plus pyrimethamine versus praziquantel for treatment of Schistosoma mansoni in Kenyan children: an open-lable, randomised controlled trial. Lancet Infect Dis., 10: 603-611.

Ojurongbe O, Adegbayi AM, Bolaji O, Akindele AA, Adefioye OA, Adeyeba OA. 2011. Asymptomatic falciparum malaria and intestinal helminths coinfection among school children in Osogbo, Nigeria. J. Res. Med. Sci., 16(5): 680-686.

Okoli CG, Anosike JC, Iwuala, MOE, Marsh K, Kinyanjui S. 2006. Immune effector mechanisms in malaria. Prevalence and Distribution of Urinary schistosomiasis in Ohaji/Egbema Local Government Area of Imo State, Nigeria Parasite Immunol., 28(1-2): 51-60.

Ologunde CA, Olaoye AB, Olaifa OA, Olowu OY. 2012. Schistosomiasis in Ogbese-Ekiti, Re-Infection After Successful Treatment with Praziquantel. Global Journal of Medical Research, 12(3): 31-36.
Rutagwera DG, Tylleskär T. 2012. Coinfection with Malaria, Hookworm and Schistosomiasis among School Children in Zambezi: A School-based Rapid Survey. Medical Journal of Zambia, 39(4): 18-23.

Snow RW, Guerra CA, Noor AM, Myint HY, Hay SI. 2005. The global distribution of clinical episodes of Plasmodium falciparum malaria. Nature, 434(7030): 214-217.

Song Y, Xiao S, Wu W, Zhang S, Xie H, Xu X, Hu X, Cui Q, Chen M, Zheng J. 1998. Preventive effect of artemether on schistosome infection. Chin. Med. J. (Engl)., 111(5): 465.

Suthar MN, Mevada AK, Pandya NH, Desai FS, Patel V, Goswami T, A comparative study of blood smear, QBC and antigen based rapid diagnostic test for diagnosis of malaria. International Journal of Biomedical And. Advance Research, 04: 222-226.

Utzinger J, N'Goran NK, N'Dri A, Lengeler C, Xiao S, Tanner M. 2000. Oral artemether for prevention of Schistosoma mansoni infection: randomised controlled trial. Lancet, 355: 1320-1325.

Xiao SH, Catto BA. 1989. In vitro and in vivo studies of the effect of artemether on Schistosoma mansoni. Antimicrob Agents Chemother., 33(9): 1557-1562.

William S, Botros S, Ismail M. 2001. Praziquantel-induced tegumental damage in vitro is diminished in schistosomes derived from praziquantel-resistant infections. Parasitology, 1: 63-66. 